\title{
Estado del arte de redes educativas para el intercambio de conocimientos en robótica educativa
}

State of the art of educational networks for the exchange of knowledge in educational robotics.

Fredy Alexander Guasmayan Guasmayan ${ }^{1}$

Nestor Andres Gonzalez Vargas ${ }^{2}$

Jose Camilo Eraso Guerrero ${ }^{3}$

Fecha de recepción: 15 de junio de 2019

Fecha de aceptación: 16 de julio de 2019

(C) 2019. Universidad de Cordoba. Este es un artículo de acceso abierto distribuido bajo los términos de la licencia de uso y distribución Creative Commons Reconocimiento 4.0 Internacional (CC BY 4.0).

\section{RESUMEN}

En este documento y para tener una idea clara de la robótica educativa, se enseñan de forma integrada y en un marco coherente, diferentes términos y conceptos. Además se presenta una exposición del estado del arte haciendo énfasis en redes educativas para el intercambio de conocimientos en el área de la robótica educativa que han sido validados en el contexto Iberoamericano. Además se describe la metodología a considerar para la implementación de una red educativa, en el contexto del proyecto "Implementación de una red para compartir material educativo en la subregión centro del Departamento del Cauca". Y por último se describe la metodología a considerar para la implementación de una estrategia de apropiación de conocimiento por parte de estudiantes universitarios utilizando rúbricas y objetos de aprendizaje.

PALABRAS CLAVE: Redes robótica educativa; Apropiación conocimiento; Teoría de control.

\begin{abstract}
In this document and to have a clear idea of educational robotics, different terms and concepts are taught in an integrated manner and in a coherent framework. In addition, an exhibition of the state of the art is presented emphasizing educational networks for the exchange of knowledge in the area of educational robotics that have been validated in the Ibero-American context. In addition, the methodology to be considered for the implementation of an educational network is described, in the context of the project "Implementation of a network to share educational material in the central subregion of the Department of Cauca". And finally, the methodology to be considered for the implementation of a knowledge appropriation strategy by university students using rubrics and learning objects is described.
\end{abstract}

${ }_{1}$ Ph.D (c) en Ciencias de la Educación. Profesor del programa de Ingeniería Mecatrónica, Universidad Mariana. Colombia. Correo: fguasmayan@umariana.edu.co

${ }^{2}$ Ph.D en Automatización Avanzada y Robótica. Profesor hora cátedra de la Facultad de Ingeniería Electrónica y Telecomunicaciones, Universidad del Cauca. Colombia. Correo: nestorgv@unicauca.edu.co

${ }^{3}$ M.Sc (c) en Automática, Universidad del Cauca. Colombia. Correo: joseeraso@unicauca.edu.co 
KEYWORDS: Redes robótica educativa; Apropiación conocimiento; Teoría de control.

\section{INTRODUCCIÓN}

La robótica educativa se ha venido popularizando en el entorno académico colombiano en los últimos años y ha ido adquiriendo un estatus propio. Por ejemplo, en el Departamento de Cundinamarca en Colombia se ejecutó el programa Lego Education (2011) para el mejoramiento de la calidad de la educación básica, secundaria y media. Tal tendencia se ha dado en diferentes regiones colombianas, y en la actualidad en las Instituciones Educativas "la robótica educativa" se ha ganado su propio espacio.

En el proyecto "Red para compartir material educativo en la subregión centro del Departamento del Cauca", se busca crear una red para promover el intercambio de conocimientos en el área de la ciencia y la tecnología, compartiendo material de robótica educativa y programación en el Programa de Ingeniería en Automática Industrial (PIAI) de la Universidad del Cauca y en Instituciones Educativas Públicas (IEP) del Cauca.

Se justifica la existencia del proyecto Red E\&C en el Departamento del Cauca, teniendo en cuenta que la robótica educativa no ha llegado a todas las IEP. Por otra parte, se ve necesario promover el aprendizaje de las bases de la robótica como medio para fomentar en los alumnos el desarrollo de conocimientos en ciencia, tecnología, ingeniería y matemáticas.

Al realizar una revisión del estado del arte de la robótica educativa se encontró un caso de éxito en España. Es el caso de los centros de primaria y secundaria del País Vasco, en el que Badiola (2013) propuso la creación de la Red Experimentar y Compartir. La red que se implementó es de bajo costo y beneficia a quince centros educativos.

Este documento está organizado de la siguiente forma: en la sección 2 se realiza una conceptualización en la que se define el término robótica educativa. En la sección 3 se describe el estado del arte de las redes educativas para el intercambio de conocimientos en el área de la robótica educativa. En la sección 4 se presenta el análisis del estado del arte. En la sección 5 se introduce la metodología a considerar para la implementación de una red educativa. En la sección 6 se expone la metodología a considerar para la implementación de una estrategia de apropiación de conocimiento en estudiantes universitarios. Y por último en la sección 6 se presentan las conclusiones, contribuciones y una idea de trabajo futuro.

\section{Conceptualización}

En esta sección y a partir de los conceptos de robótica y educación, se presenta el de robótica educativa. .

\subsection{Robótica}

Según Barrientos et al. (2007) la robótica posee un reconocido carácter interdisciplinario, participando en ella diferentes disciplinas básicas y tecnologías tales como la teoría de control, la mecánica, la electrónica, el álgebra y la informática entre otras.

\subsection{Educación}

El término educación ha inquietado a muchos autores, por lo que existen varias definiciones. Según la UNESCO (2011) la educación es el proceso mediante el cual las sociedades transmiten en forma intencionada el acervo de información, comprensión, conocimientos, actitudes, valores, habilidades, competencias y comportamientos de una generación a otra. Comprende el uso de actividades de comunicación destinadas a producir aprendizaje.

\subsection{Robótica Educativa}

Veamos a continuación algunas de las definiciones de la robótica educativa.

Para Acuña y Lourdes (2012) la robótica educativa promueve en el alumno una serie de beneficios, conjunto de desempeños y habilidades que se ven directamente reflejados en el crecimiento de la creatividad, el diseño, la construcción, la programación y divulgación de creaciones propias. Márquez y Ruiz (2014) plantean la robótica educativa como una metodología de aprendizaje que se ha desarrollado como una opción de solución de problemas derivados de distintas áreas del conocimiento como las matemáticas, las ciencias naturales y experimentales, la tecnología, las ciencias de la información y la comunicación. Chitolina et al. (2016) muestra una definición puntual de robótica educativa, catalogándola como una práctica en la que se pone en funcionamiento 
la unión del hardware y el software, esto con el fin de diseñar y desarrollar robots que apoyen a estudiantes en el inicio de estudios de las ciencias y la tecnología.

La robótica educativa es, en resumen, es el conjunto de actividades pedagógicas que desarrollan habilidades de pensamiento en el estudiante, a través de la construcción y programación de robots.

\section{Estado del arte de las redes de robótica educativa}

REn este apartado se describe el estado del arte de las redes de robótica educativa en el contexto internacional, nacional y local.

\subsection{Contexto internacional}

La robótica educativa es una tecnología que se apoya en diferentes disciplinas, y es la herramienta perfecta para enseñar diferentes áreas como la informática. Es por esto que en el contexto internacional se está incorporando la robótica como estrategia pedagógica en los centros educativos. Una detallada revisión de la robótica educativa puede encontrarse en el trabajo de Benitti (2012) y Herrera y Rincón (2013).

En el trabajo de Balch et al. (2008) se recopiló la experiencia del Tecnológico de Georgia. Este trabajo muestra cómo enfrentaron un problema de desinterés por parte de los jóvenes que estudiaban ciencias de la computación. Introdujeron la robótica en el curso inicial, suministraron robots educativos a los alumnos y se propuso un enfoque de implementación de tareas de "piso bajo y techo alto", es decir, lo suficientemente complejas para novatos pero también interesantes para ser desarrolladas por expertos. La implementación de este enfoque arrojó excelentes resultados y fue bien acogida por los alumnos.

El problema del desinterés de los jóvenes por las ciencias de la computación no solo se presenta en la educación universitaria. Blank (2006) realizó una investigación con alumnos de la escuela secundaria en Pensilvania donde un grupo de alumnos utilizaron el laboratorio de robótica y aprendieron a usar el programa informático Myro. Lo anterior con el fin de dar una visión más divertida y motivadora a los jóvenes para que estudiaran ciencias de la computación. Los resultados de la investigación confirmaron que a través de la robótica, la programación se puede tornar interesante e incluso entretenida para los alumnos.

En el caso de centros de primaria y secundaria, Badiola (2013) propuso la creación de la Red Experimentar y Compartir, para centros educativos del País Vasco (España). El proyecto que costó alrededor de tres mil euros, beneficia a quince centros educativos y está destinado al aprendizaje de las bases de la robótica como medio para fomentar en los alumnos el desarrollo de conocimientos en ciencia, tecnología, ingeniería y matemáticas. Además se ha comprobado que mejora sus habilidades comunicativas, de lectoescritura, trabajo en equipo y resolución de problemas.

Pero no solo en la educación presencial se han creado estrategias de aprendizaje basadas en la robótica y automatización educativa. En la educación virtual también se han buscado estrategias para incentivar en los alumnos la generación de conocimiento a partir de la programación de sistemas virtuales. Por ejemplo, en el trabajo de George y Despres (1999), se recopila la experiencia de varios docentes que diseñaron una metodología de enseñanza virtual soportada por videoconferencias, video tutoriales y correos electrónicos. La plataforma informática utilizada para la programación y la visualización del robot se llama Roboteach. Dicha plataforma permite a los alumnos manipular el robot y crear un programa de control.

\subsection{Contexto nacional}

En el contexto nacional existen concursos de robótica en los que participan alumnos de colegio y universitarios. Se cuenta por ejemplo con el FIRST LEGO LEAGE que es para jóvenes de entre 8 y 14 años, y que selecciona el representante de Colombia para el concurso mundial. Otro certamen con presencia en Colombia es el VEX robotic competitions, en el que la ingeniería y la construcción de robots móviles son los protagonistas. Debido a su escasa difusión estos concursos consiguen despertar el interés de un grupo muy reducido de alumnos de robótica y programación. Su carácter anual tampoco ayuda.

En el Departamento de Nariño se obtuvo una experiencia valiosa a través de la robótica educativa 
en la universidad e instituciones educativas, con el programa financiado por el Sistema General de Regalías y que se titula "Desarrollo y Promoción de competencias científicas y tecnológicas en Robótica e Informática en la Universidad de Nariño y establecimientos educativos del municipio de Pasto"

Según Montes (2008) el país está lejos de una utilización intensa de la robótica, como ocurre por ejemplo en Japón o en Estados Unidos. Aunque hay investigación, no es suficiente como para estimular la producción de este tipo de tecnologías en centros académicos e industriales.

\subsection{Contexto local}

En algunas Instituciones Educativas y Universidades del Departamento del Cauca la robótica educativa ha incursionado. Por ejemplo en la ciudad de Popayán en el Colegio Campestre Americano existe un club de robótica y en la Universidad Antonio Nariño y en la Universidad del Cauca cuentan con semilleros de robótica.

A nivel formativo existe una empresa en Popayán que se llama DEMOSS y que ofrece cursos de robótica educativa y impresión $3 \mathrm{D}$. Adicionalmente en el Servicio Nacional de Aprendizaje SENA, se imparten formaciones tecnológicas que contemplan la automatización y la mecatrónica.

\section{Análisis del estado del arte}

Se ha realizado una revisión y análisis de los artículos de robótica educativa, logrando ofrecer una idea sobre el panorama actual de esta temática. De los trabajos revisados se selecciona como principal referente, la propuesta de la Red Experimentar y Compartir del País Vasco (Badiola, 2013), ya que se ha implementado con éxito en la educación primaria y secundaria. A nivel universitario se escoge como referente la propuesta del Tecnológico de Georgia (Balch et al, 2008). Realizar algo similar en las instituciones educativas de la subregión centro del Departamento del Cauca tendría un costo muy reducido.

\section{Metodología a considerar para la implementación de una red educativa}

A continuación se relacionan las fases propuestas para conformar la red tras la elaboración del estado del arte:
Fase 1. Diseñar la página web que soportará la red experimenta y comparte (Red E\&C) del Cauca. Se encuentra disponible el dominio: http://www. unicauca.edu.co/eyc/

Fase 2. Formación de profesores y lanzamiento de la Red E\&C.

Fase 3. Visitas a los salones de clase y validación de la formación en la universidad y en los colegios.

Fase 4. Análisis de resultados y redacción de la memoria que recopile la experiencia de implementación de la Red E\&C.

Fase 5. Servicio de soporte de la página web y formulación de un nuevo proyecto para ampliar la Red E\&C a otras regiones.

\section{Metodología a considerar para la implementación de una estrategia de apropiación de conocimiento}

A través de la Red E\&C se propone la implementación de una estrategia de apropiación de conocimiento de la teoría de control que utiliza rúbricas de Lego Education y objetos de aprendizaje como tabletas y sets de Lego Mindstorms EV3. Además se plantea poner a prueba la metodología con los estudiantes de Ingeniería Mecatrónica de la Universidad Mariana. Los estudiantes cumplen con unos itinerarios de formación en donde se les introduce a la teoría de control y con un enfoque orientado a proyectos se les propone que construyan y programen robots.

\section{Conclusiones}

- $\quad$ Se ha realizado una revisión y análisis de trabajos publicados hasta el año 2018, logrando ofrecer una idea sobre el panorama actual de las redes educativas que intercambian conocimientos. Además se han recopilado experiencias representativas en el contexto internacional, nacional y local en el ámbito de la Robótica Educativa. En el trabajo se ha resaltado las propuestas de Balch et al. (2008) en Estados Unidos y Badiola (2013) en España, porque se han implementado con éxito en la universidad y en la educación primaria y secundaria, respectivamente. Realizar algo similar en las instituciones educativas de la subregión centro del Departamento del Cauca tendría un costo muy reducido. Por ejemplo, la red 
educativa de Badiola (2013) se implementó con alrededor de tres mil euros y beneficia a quince centros educativos.

- $\quad$ El problema del desinterés de los jóvenes por la ciencia y tecnología se puede combatir a través de la Robótica Educativa, ya que esta última le puede dar una visión más divertida y motivadora a los jóvenes para que estudien carreras afines.

\section{Agradecimientos}

Este trabajo fue desarrollado gracias a la financiación conjunta de la Universidad del Cauca y el Programa InnovAcción Cauca (Convocatoria 05-2015 de Inserción de Doctores)

\section{REFERENCIAS}

[1]. Acuña, A. y Lourdes, A. (2012). Diseño y administración de proyectos de robótica educativa: Lecciones aprendidas. Teoría de la Educación. Educación y Cultura en la Sociedad de la Información, 13(3), 6-27.

[2].Badiola, M. (2013). Red experimentar y compartir. Recuperado el 6 de agosto de 2018, de https://sites.google.com/site/ redikaskide/

[3].Balch, T., Summet, J., Blank, D., Kumar, D., Guzdial, M., O'Hara, K., Gavin, A. (2008). Designing personal robots for education: hardware, software and curriculum. Pervasive Computing, 7(2), 5-9.

[4].Barrientos, A., Peñin, L., Balaguer, C., Aracil, R. (2007). Fundamentos de robótica. España, Madrid: McGraw-Hill Interamericana de España.

[5].Benitti, F. (2012). Exploring the educational potential of robotics in schools: A systematic review. Computers \& Education, 58(3), 978-988.

[6].Blank, D. (2006). Robots make computer science personal. Communications of the ACM, 49(12), 25-27.

[7].Centro virtual de noticias de la educación. (2011). Programa Lego education en
Cundinamarca. Recuperado el 22 de junio de 2016, de http://www.mineducacion.gov. co/cvn/1665/w3-article-274057.html

[8].Chitolina, R., Noronha, F., Backes L. (2016). A Robótica Educacional como tecnologia potencializadora da aprendizagem: das ciências da natureza às ciências da computação. Educação, Formação \& Tecnologias. 9 (2), 56-65.

[9].George, S. y Despres, C. (1999). A multi-agent system for a distance support in educational robotics. International Conference on Telecommunication for Education and Training, 344-353.

[10].Herrera, C. y Rincón, D. (2013). Estado del arte de la robótica educativa en el ámbito mundial. Bogotá: Repositorio Institucional de la Corporación Universitaria Minuto de Dios.

[11].Márquez, J. y Ruiz, J. (2014). Robótica educativa aplicada a la enseñanza básica secundaria. Didáctica, Innovación y Multimedia, 10(30), 1-12

[12].Montes, A. (2008). Robótica colombiana. Revista Semana. Recuperado el 15 de enero de 2017, de http://www.semana. com/vida-moderna/articulo/roboticacolombiana/92865-3

[13].UNESCO (2011). Clasificación Internacional Normalizada de la Educación: CINE-2011. 